7

8

9

10

This is a non-peer reviewed preprint submitted to EarthArXiv.

12

13

14

15

16

17

18

19 


\title{
Two Pixel Reference Algorithm
}

\author{
Ziheng Sun ${ }^{1}$, Liping $\mathrm{Di}^{1}$, Hui Fang ${ }^{1}$ \\ zsun@gmu.edu; 1di@gmu.edu (corresponding author); hfang1288@gmail.com \\ ${ }^{1}$ Center for Spatial Information Science and Systems (CSISS) \\ George Mason University \\ 4087 University Dr STE 3100 \\ Fairfax, VA, USA
}

Abstract: Object based image analysis (OBIA) has a unique process requirement: relate all the pixels in the segmented images to the vectorized polygons (pixel in polygon). The existing solutions are very slow in finding the pixels in a polygon. This paper proposes a novel algorithm called Two-Pixel-Reference to speed up the process. The algorithm is initially designed for segmented remote sensing images. It avoids most multiple-layer loops in existing methods and trims many redundant comparison among pixels. Thus it has literal lower Big $\mathrm{O}$ algorithm complexity. We implemented the algorithms in $\mathrm{C}++$ and made more than seventy tests on two different machines to compare the algorithm with three other existing algorithms. The results show that it significantly decreases the time cost of the process. In every single test, the proposed algorithm costs much less time than other algorithms. Specifically, the average duration is reduced from 3.96 seconds to 0.15 second on machine \#1 and from 3.647 seconds to 0.073 second on machine \#2. This paper makes a good example for researching time-efficient algorithms to accelerate the overall process of OBIA in such a big data era. 
43 Keywords: object based image analysis; pixel in polygon; complexity; remote sensing; image

44 classification; vectorization.

45

46 


\section{Introduction}

Object based image analysis (OBIA) is one of the state-of-the-art techniques in RS [1-6].

It first segments an image into objects, which are also called patches, segments or regions.

Then the post processes are conducted on the objects rather than pixels (as shown in Fig. 1).

Many study cases have proven that OBIA is more advantageous on enhancing the classification accuracy than pixel based image analysis (PBIA) [7-10]. However, due to the new object layer, OBIA has more steps than PBIA and the overall time cost of OBIA is generally higher. More efforts on speeding up OBIA are needed to increase the time value of

OBIA results.

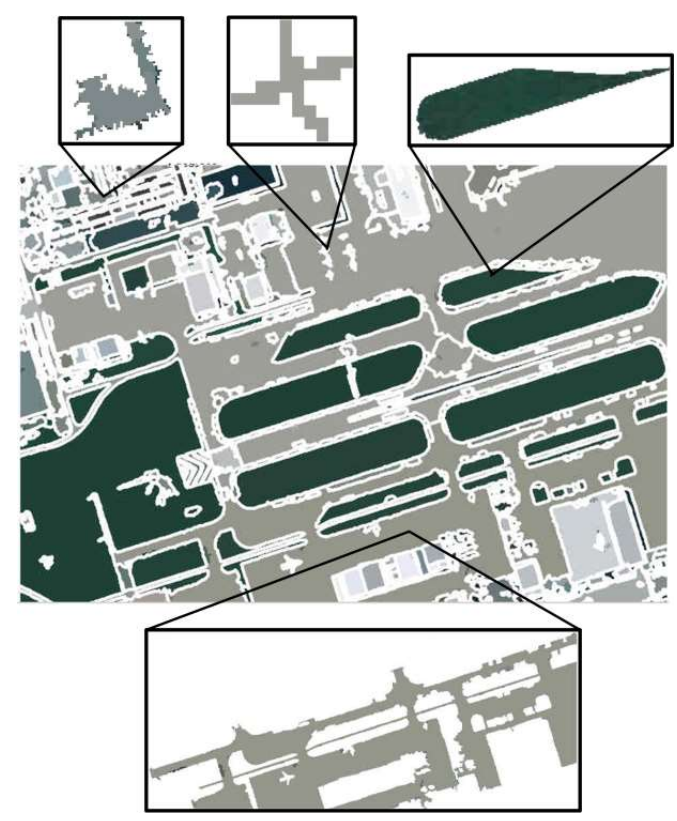

Figure 1. A segmented RS image and some sample segments

According to our experiences in building and using OBIA system $[3,11]$, a large block of time of an OBIA analysis is spent on sorting pixels in each region after an image is segmented. The existing methods have high time complexity and are very inefficient for time saving purpose. Thus, this paper proposes a new algorithm of sorting pixels to decrease the duration 
into a senseless level. The algorithm avoids most multiple-layer loops in existing methods and trims many redundant comparison among pixels. Finally it evolves into an extremely concise method with very low time complexity.

To test the algorithm, we compared four algorithms, including three existing algorithms, on two machines. The inputs include an image and a vector containing polygons generated from the image by raster-to-vector conversion. The output is an image, in which each pixel value is set as the identifier of the vector feature the pixel belongs to. In all the experiments, the input files and the output files are exactly the same. The only difference is the duration of algorithm execution. We recorded the execution time and made an analysis. The results show that the proposed algorithm significantly decrease the time cost. Specifically, the average duration is reduced from 3.96 seconds to 0.15 second on machine \#1 and from 3.647 seconds to 0.073 second on machine \#2.

The remainder of this paper is organized as follows. Section 2 introduces the background knowledge. Section 3 details the new algorithm and three existing algorithms. In section 4 the experiments are described and the results are evaluated. Section 5 discusses our original contribution to the community. Section 6 concludes the paper and gives the future work.

\section{Background}

A large amount of RS data has been obtained by satellite-based or in-situ (e.g., airborne) sensors in the last few decades. Today the amount is still aggressively growing [12, 13]. For example, the NASA Earth Observing System Data and Information System (EOSDIS) [14] has archived over 3.5 million individual Landsat scenes totaling around 1 petabyte of imagery $[15,16]$. There are many other RS products and data archive centers around the world. 
Comparing to the speed of obtaining RS images, the speed of processing and analyzing images is relatively slow $[17,18]$. The complexity of applied algorithms, especially time complexity, has big influences on the speed, and certainly the time value of the information extracted from RS images [19]. Lowering algorithm complexity can reduce the time cost of image analysis and enhance our capability of timely discovering valuable information within the huge RS data.

OBIA, one of the current researched hot topics in RS, is comprised of three major steps: image segmentation, post process and object analysis [20] (as shown in Fig. 2). Each step has a set of algorithm choices. Image segmentation filters RS images into a collection of regions [21]. The pixels in one region have the same value. The algorithms for this step have k-means [22], ISODATA [23], mean shift [24], etc. The segmented image goes through a post process including band combination, vectorizing and pixel sorting. The band combination process merges all the bands into only one band. To ensure the mappings between image objects and vector features are bijection, the combination should be reversible which means the one band can be decomposed back to the original bands. The one band is then vectorized into a vector file which outlines the image objects with polygons. Each polygon is managed as a vector feature in geographic information systems. A sorting process will take the segmented image and the vector features as inputs, figure out the containing relationships between the vector features and the image pixels and output a image in which each pixel's value is set as the identifier of the feature it belongs to (Fig. 3).

The post process is essential for the next process in the third step such as calculating the properties of image objects. The properties of image objects have three major categories: 

[28-30].
111

spatial, spectral and texture [25-27]. The spectral and texture properties of a feature are calculated based on the original values of the contained pixels. Once the property values are generated, people will be able to do some object analysis on them, e.g., supervised classification or feature extraction (buildings, rivers, roads, forests, lakes, lawns, planes, etc)

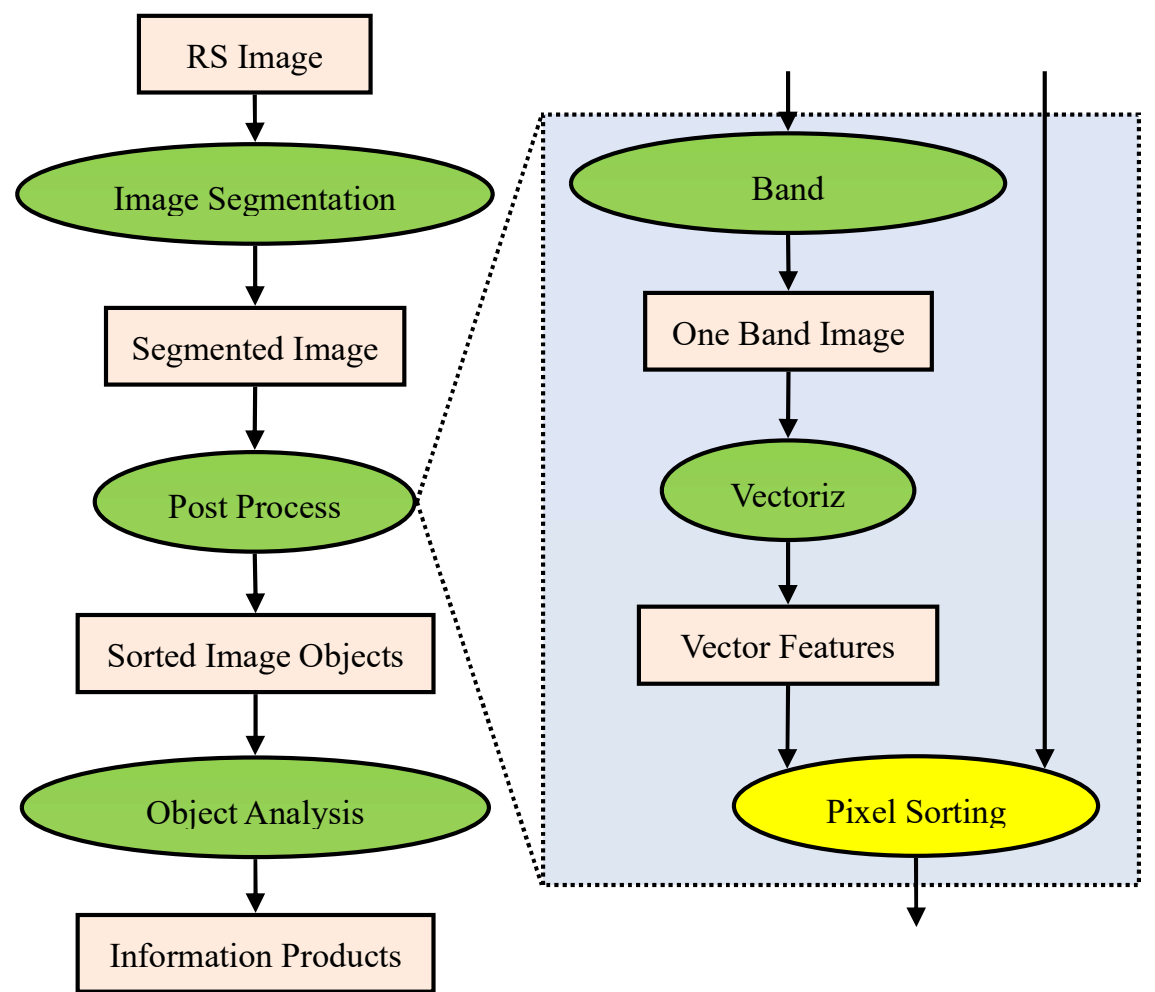

Figure 2. The workflow of OBIA 

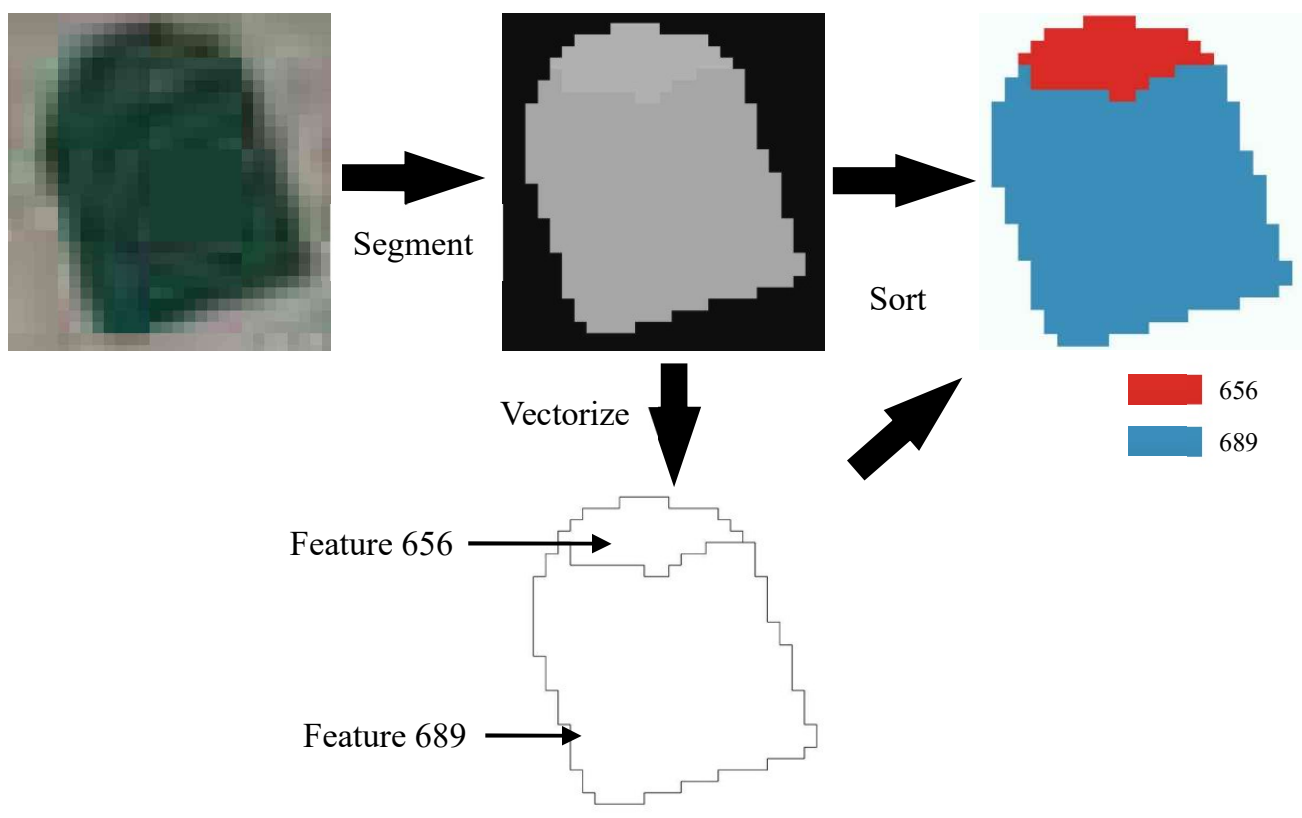

656

Figure 3. Sorting pixels located in vectorized polygons

\section{Algorithms}

This section will first list three existing algorithms as comparison and then introduce a new algorithm called two-pixel-reference algorithm. All the algorithms are able to complete the pixel sorting task. But their algorithm complexity is different.

\subsection{Shared parameters and variables}

Given the inputted image is a one-band image containing $\boldsymbol{X}$ columns and $\boldsymbol{Y}$ rows of pixels. The value of the pixel at column $\boldsymbol{x}$ and row $\boldsymbol{y}$ is represented by function $\boldsymbol{F}_{(x, y)}$. The value of variable $\boldsymbol{x}$ ranges from 1 to $\boldsymbol{X}$. The value of variable $\boldsymbol{y}$ ranges from 1 to $\boldsymbol{Y}$. The corresponding inputted vector file has $\boldsymbol{M}$ features/polygons whose identifiers are assigned in sequence from 1 to $\boldsymbol{M}$. The features are represented by $\boldsymbol{V} \boldsymbol{F}$. 


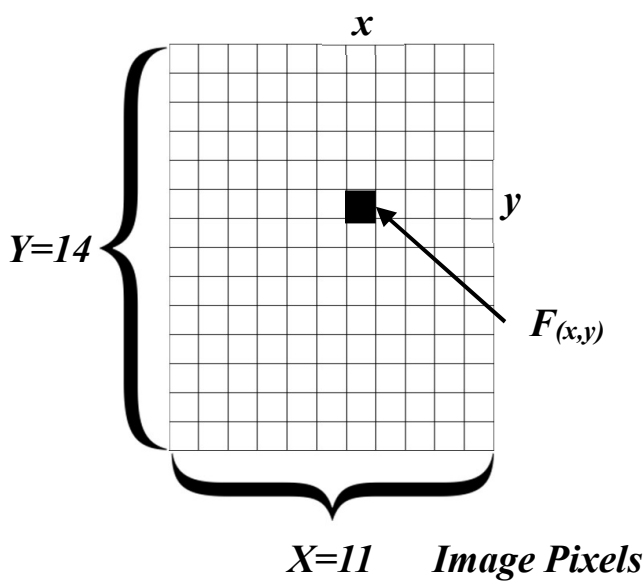

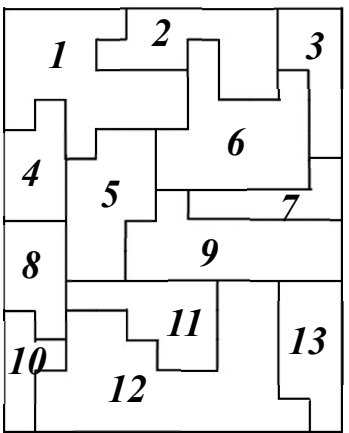

Vector Features
125

Figure 4. Example inputted image and vector parameters and variables

\subsection{Existing algorithms}

1) Algorithm \#1: This algorithm is based on the widely used four direction seed spreading algorithm. The basic idea is finding a pixel located in a feature and adding it as a seed into a list. Then iterate all its neighbor pixels to find if there is any pixels belonging to the same feature. Four direction means only the pixels located on the east, west, south and north are considered as neighbor pixels. If a neighbor pixel is identified within the feature, it will be taken as a new seed and added to the list. The spreading process is repeated on the new seeds until all the pixels in the list have been processed. Finally, the list will contain all the pixels within the feature. The core steps of this algorithm are detailed below.

1. Get the first seed pixel $\boldsymbol{S P}_{\boldsymbol{i}}$ for each feature $\boldsymbol{f}_{\boldsymbol{i}}$ in $\boldsymbol{V F}$.

2. For each feature $\boldsymbol{f i}$, add its seed $\boldsymbol{S} \boldsymbol{P}_{\boldsymbol{i}}$ to list $\boldsymbol{L}$.

3. Compare $\boldsymbol{S} \boldsymbol{P}_{\boldsymbol{i}}$ with its four neighbors. If a neighbor has the same value with the seed, add it to $\boldsymbol{L}$. After all the neighbors are compared, mark $\boldsymbol{S} \boldsymbol{P}_{\boldsymbol{i}}$ as checked.

4. Repeat Step 3 on the unchecked seed in $\boldsymbol{L}$ until all the seeds in $\boldsymbol{L}$ are checked. The pixels in 
$\boldsymbol{L}$ will be the pixels contained in $\boldsymbol{f}_{\boldsymbol{i}}$.

5. Repeat Step 1 4 to sort all the features in $\boldsymbol{V F}$.

Because the deepest step (Step 3) in the loops could be run by up to $\mathrm{M} \times(X \times Y)^{4}$ times, the Big-O complexity of this algorithm is:

2) Algorithm \#2: This algorithm is an improved version of algorithm \#1. It takes advantage of a characteristics of the segmented image. If the first seed's pixel value of a feature is unique among the first seeds of all the features, then all the pixels with this value directly belongs to the feature. The steps of algorithm \#2 are:

1. Get the first seed pixels for all the features in $\boldsymbol{V} \boldsymbol{F}$.

2. If the first seed pixel value of $f_{i}$ is unique, find all the pixels with this value and belong them to $f_{i}$.

3. For those features whose first seed pixel values are not unique, apply Algorithm \#1. complexity of algorithm $\# 2$ is: the position of features, while this algorithm is in the perspective of pixels. The steps are described below.

1. Get the first seed pixels for all the features in $\boldsymbol{V F}$. Add them to a list $\boldsymbol{L}_{\boldsymbol{1}}$.

2. All the pixels are initially marked as unchecked. Scan the pixels line by line. 
3. For each unchecked pixel $\boldsymbol{P}(\boldsymbol{x}, \boldsymbol{y})$, use it as a seed and apply Algorithm \#1 to get a list of pixels $\boldsymbol{L}_{2}$.

4. Search $\boldsymbol{L}_{1}$ to get the first seed which is listed in $\boldsymbol{L}_{2}$. Get the feature corresponding to the seed. Belong $\boldsymbol{L}_{2}$ to the feature.

5. Mark all the pixels in $\boldsymbol{L}_{2}$ as checked.

6. Repeat 3 5 until all the pixels in the image are checked.

The complexity of this algorithm is:

$$
T_{\text {algorithm } 3}=O_{\left((X \times Y)^{4}\right)}
$$

\subsection{Two-Pixel-Reference (TPR) Algorithm}

This algorithm avoids most loops in the above three algorithms and trims redundant to ensure the complexity stays low. The steps are detailed below.

1. For each feature $\boldsymbol{f}_{\boldsymbol{i}}$ in $\boldsymbol{V F}$, mark the pixels adjacent to its boundary and locating within $\boldsymbol{f}_{\boldsymbol{i}}$ by

$f_{i}$ 's identifier. Repeat this step until all the pixels adjacent to feature boundaries are marked.

2. Scan the image row by row. If a pixel $\boldsymbol{P}_{(x, y)}$ is not marked, compare its value $\boldsymbol{F}_{(x, y)}$ with $\boldsymbol{F}_{(x-1, y)}$ and $\boldsymbol{F}_{(x, y-1)}$. If equal to one of them, mark $\boldsymbol{P}$ with the equal one's feature identifier. Repeat this step until all the pixels are marked.

3. Set the identifier values to corresponding pixels and output the image. 


$$
O_{\text {algorithm } 4}=O_{(\mathrm{X} \times \mathrm{Y})}
$$

According to the Big $\mathrm{O}$ algorithm complexity rules [31, 32], The four algorithms are

placed in order from high complexity to low complexity as:

$$
O_{\text {algorithm }}>O_{\text {algorithm } 2}>O_{\text {algorithm } 3}>O_{\text {algorithm } 4}
$$

Therefore, TPR algorithm has the lowest algorithm complexity literally. Next section will test the physical influences on time cost through experiments.

\section{Implementation, Experiment and Results}

We implement the four algorithms using $\mathrm{C}++$ language with support from GDAL library [33]. The code is available in a public GitHub repository https://github.com/VirginiaJRS/PixelsInSegment.

We run the code on a test image on two machines. Fig. 5a shows the original test image, a high resolution optical remote sensing image of the Dallas Love Field Airport at Texas, U.S.

Fig. $5 \mathrm{~b}$ and $5 \mathrm{c}$ are the input files of the algorithms and Fig. $5 \mathrm{~d}$ is the outputted image. One test machine is a HP laptop with Windows 7 64-bit system, Intel Core i5-2450M CPU (2.50GHz) and 6 gigabytes RAM memory. The other machine is a HP Proliant DL180 G6 rack server with Ubuntu Linux 12.04 LTS system, 8 core Intel(R) Xeon(R) CPU E5606 (2.13GHz) and 8 gigabytes RAM memory.

As the operating systems are in parallel processing mode, other running threads at the same time will definitely impact the duration of each execution of the algorithms. To reduce the uncertain impacts of the external factors and create a fair test environment, we killed as many threads as possible before the tests are conducted. During the test, both machines are dedicated and not be used for any other tasks. The execution time is automatically recorded 

multiple plots as shown in Fig. 6.

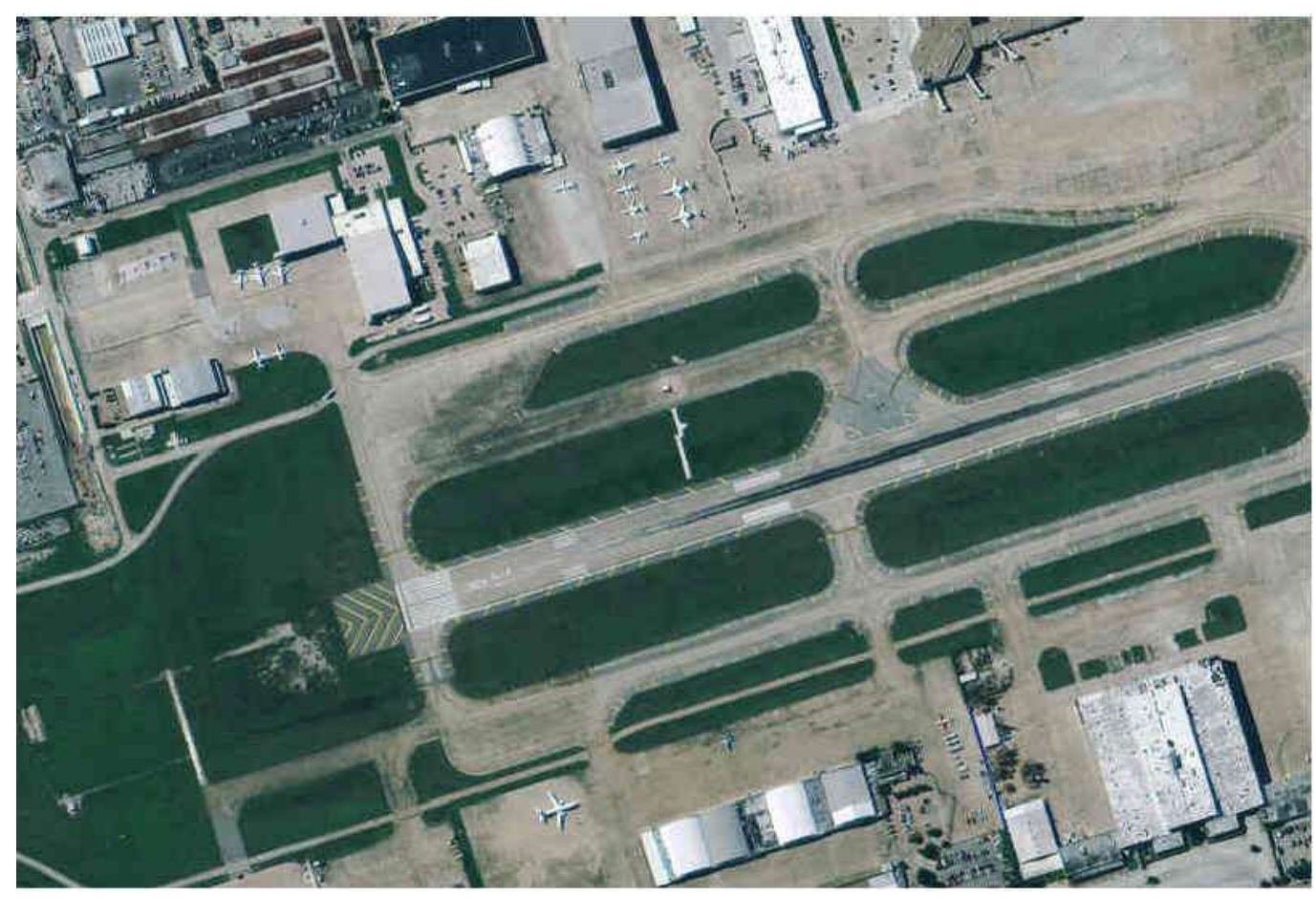

(a)

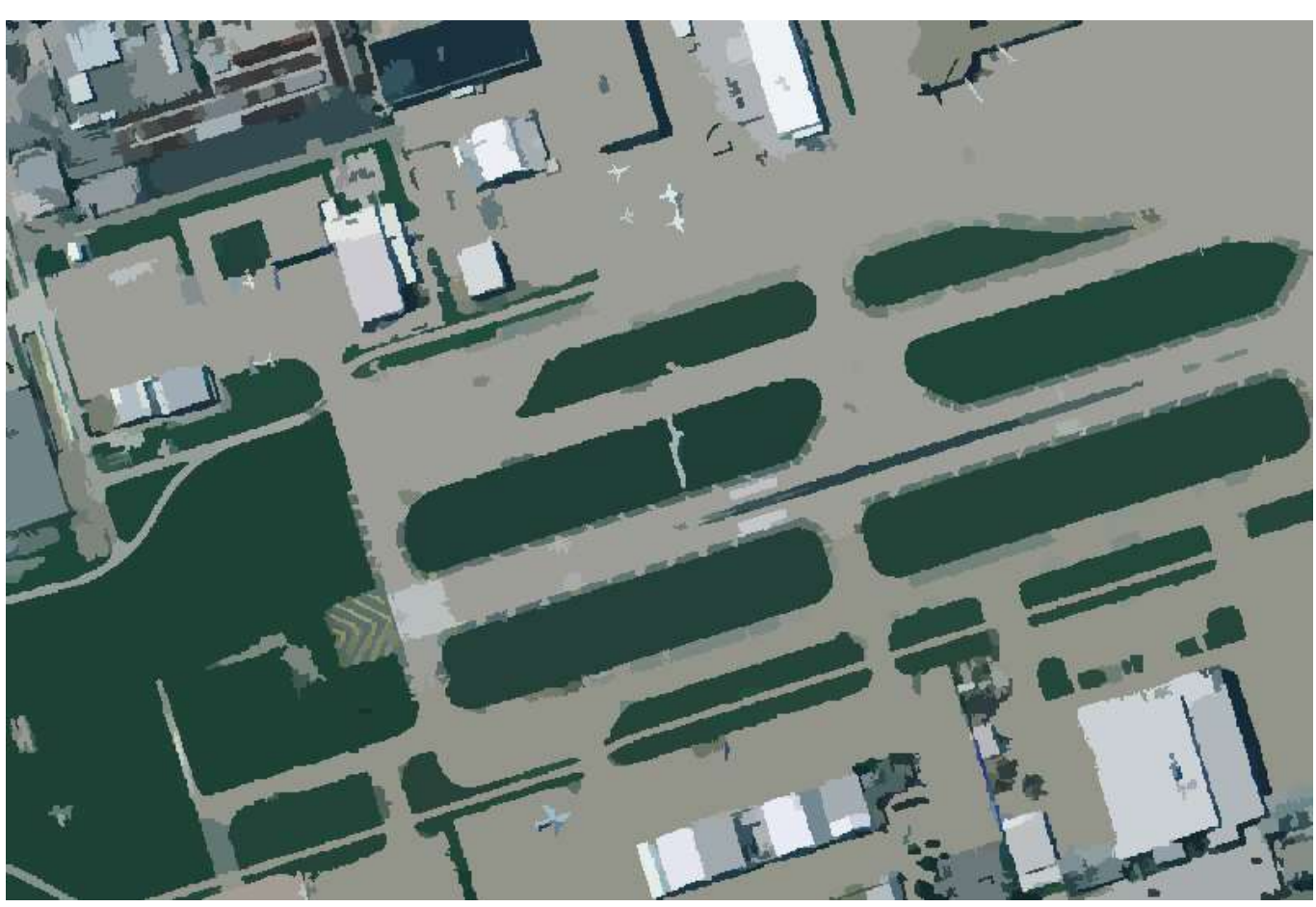




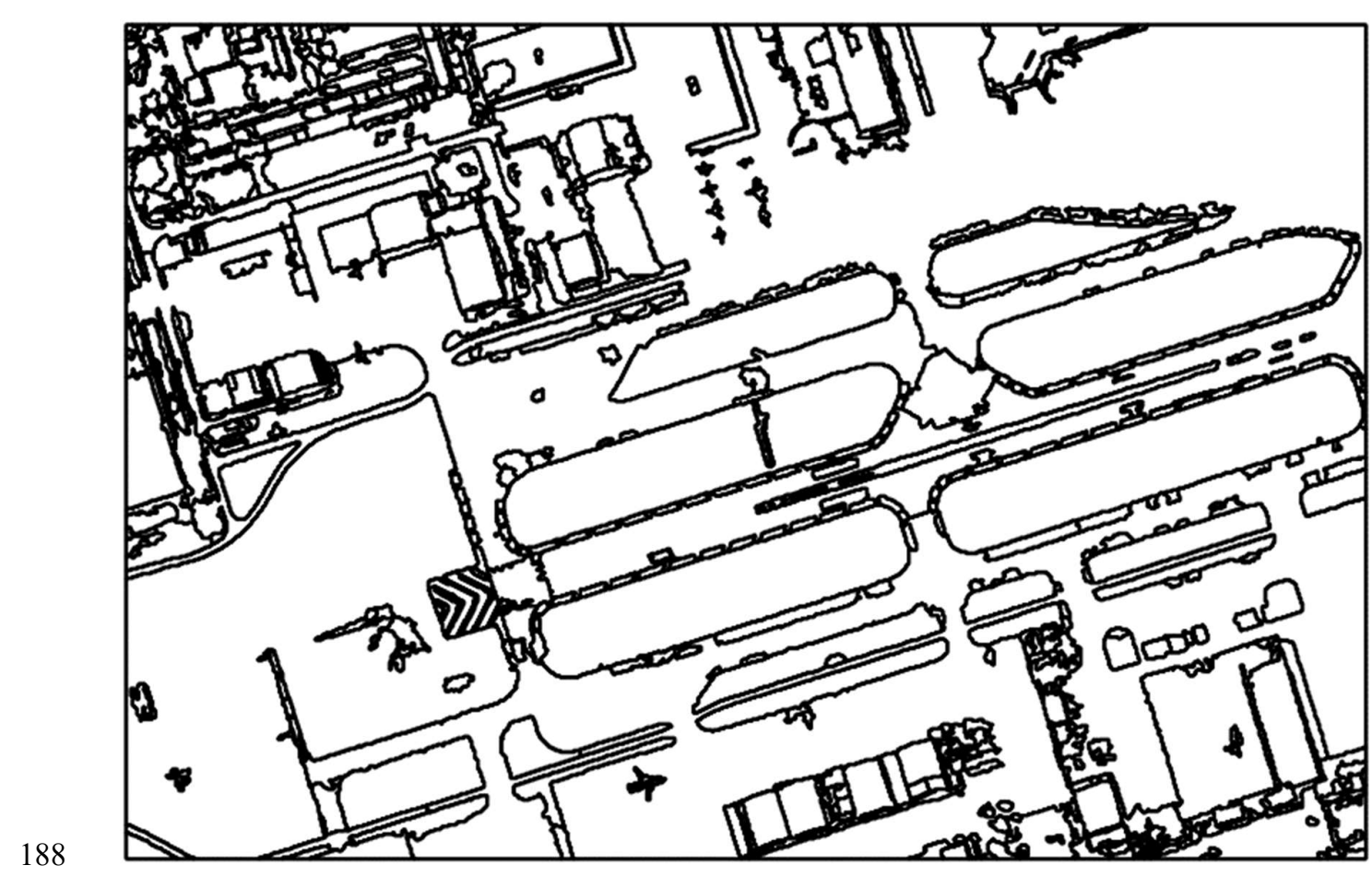

(c)

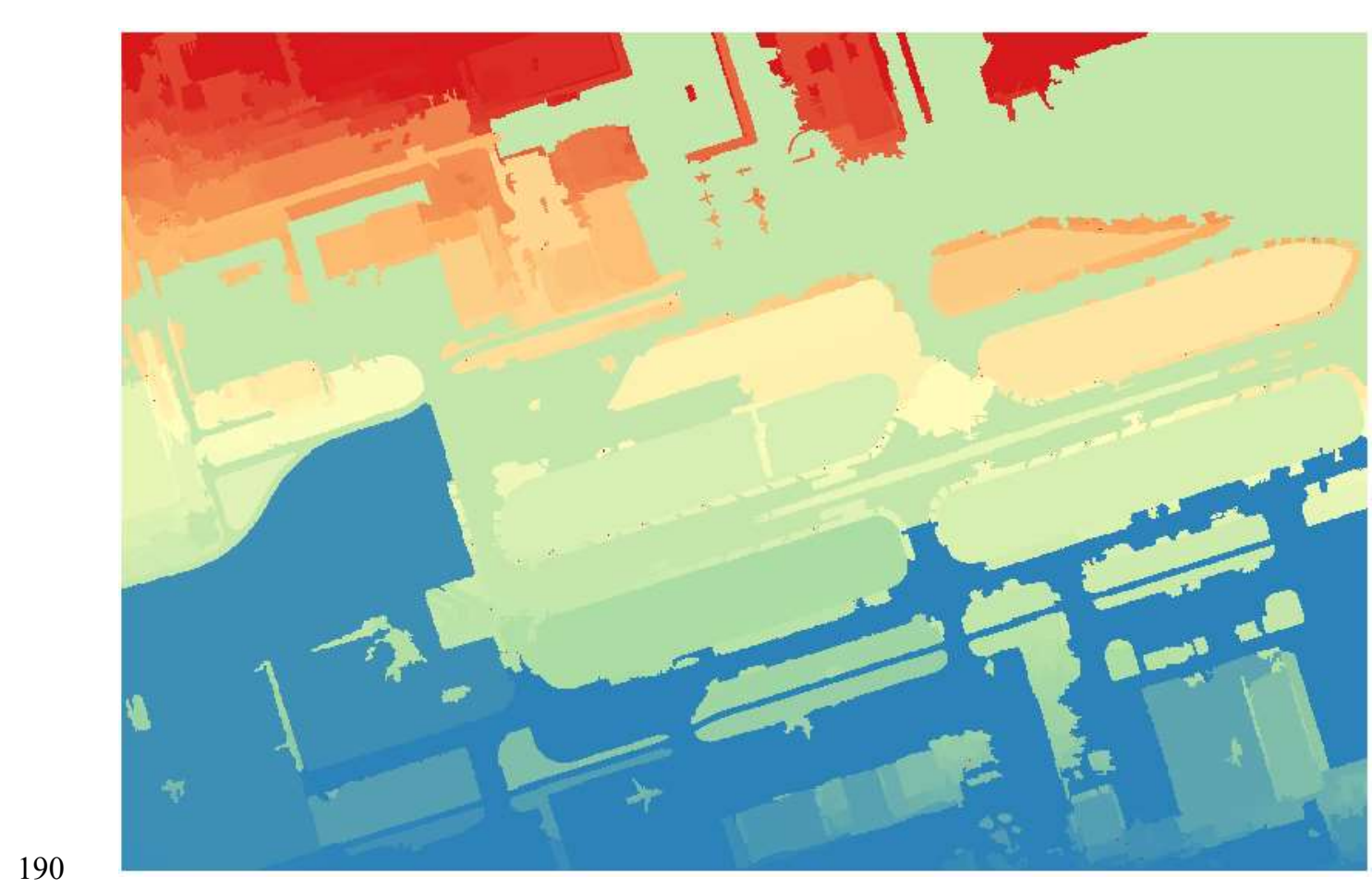

192 Figure 5. The inputs and output of the test image. (a) original image; (b) segmented image; (c) 

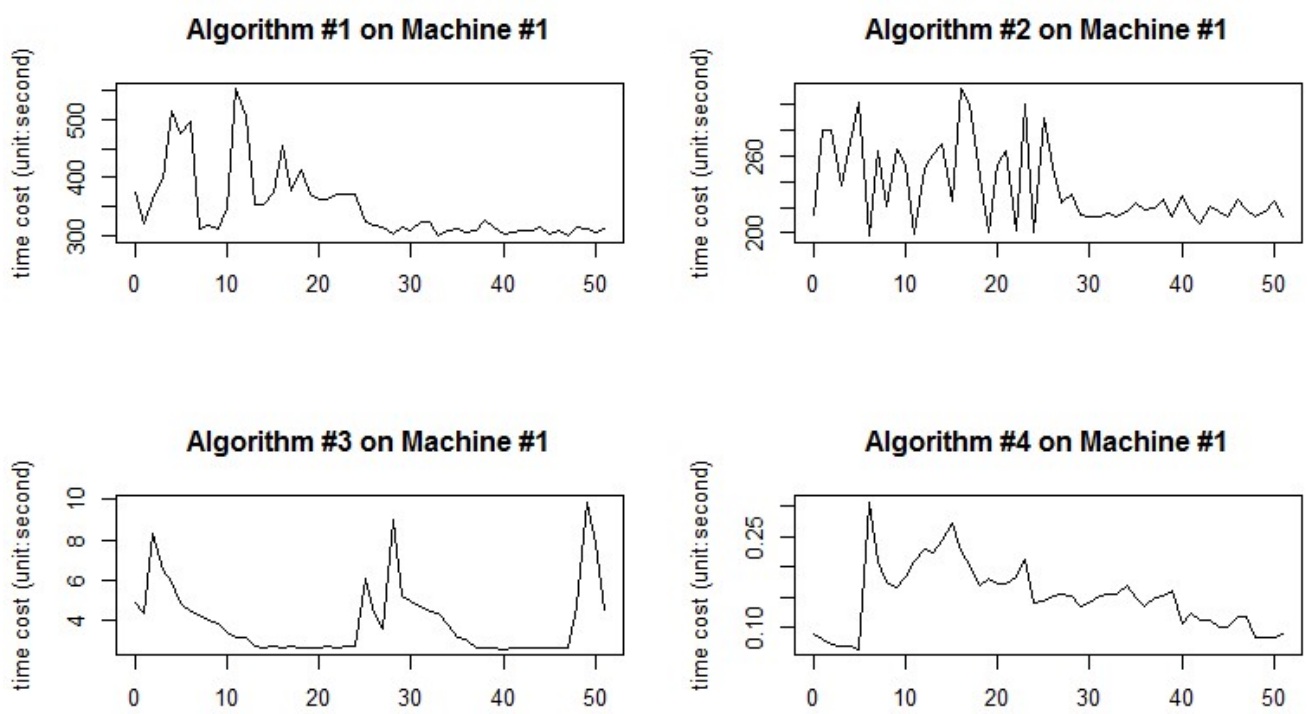

194
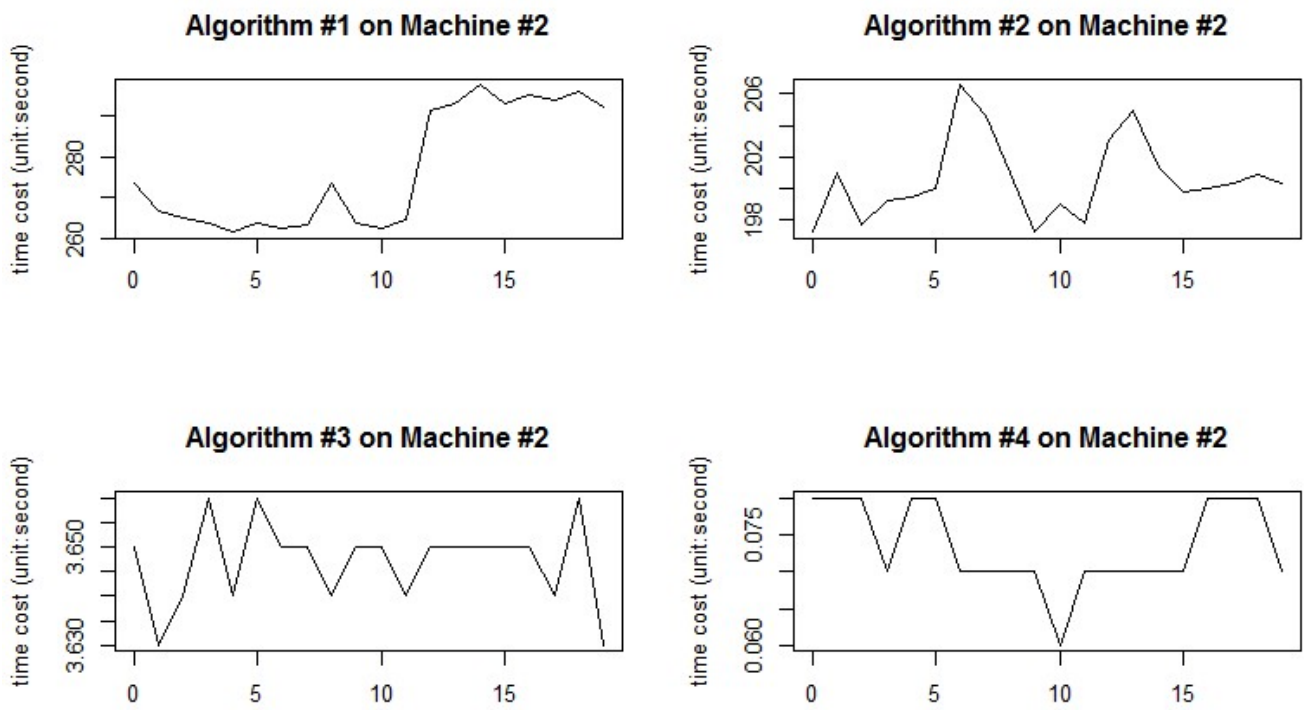

195

Figure 6. The time costs of four algorithms on two machines

In Fig. 6, overall fifty tests are recorded on machine \#1 and twenty tests are recorded on \#2. Although we tried to make a clean test environment, the time cost still varies largely for the same algorithm. For example, the time cost of Algorithm \#1 on machine \#1 ranges from 


\begin{tabular}{lllll}
\hline & Algorithm \#1 & Algorithm \#2 & Algorithm \#3 & Algorithm \#4 \\
\hline Machine \#1 & $300 \sim 550$ & $200 \sim 280$ & $2 \sim 10$ & $0.07 \sim 0.3$ \\
Machine \#2 & $260 \sim 300$ & $197 \sim 207$ & $3.63 \sim 3.66$ & $0.06 \sim 0.08$ \\
\hline
\end{tabular}

300 seconds to 550 seconds, while the time cost on machine \#2 ranges from 260 seconds to 300 seconds. Generally for all the four algorithms, the time cost on machine \#2, which is a rack server, is more stable than machine \#1 which is a laptop. But the differences on time cost among algorithms are still very apparent. The range of time cost of the four algorithms is listed in Table 1. All the ranges are never overlapped with each other and have a clear high-to-low sequence which completely agrees with the literal order analyzed in Section 3. The time cost of the TPR algorithm (\#4) is obviously the lowest on both machines. We also calculated the average time cost of each algorithm on the two machines (Table 2). The time cost of algorithm \#4 is always below 0.2 second. Such an interval is actually senseless by human beings. Comparing to the time cost of 3 seconds (algorithm \#3), 200 seconds (algorithm \#2) and 270 seconds (algorithm \#1), TPR algorithm significantly compressed the time cost of this process into a very satisfying level.

The dimension of the test image is 800 pixels $(\boldsymbol{X})$ by 538 pixels $(\boldsymbol{Y})$ and the number of features is $900(\boldsymbol{M})$. If the inputted image is changed to a larger one and the feature number also increases, the time cost of each algorithm will definitely increase. According to the Big-O algorithm complexity rules, the time cost of algorithm \#4 will remain the lowest among the four.

Table 1. The ranges of time cost for the four algorithms (unit: second) 


\begin{tabular}{lllll}
\hline & Algorithm \#1 & Algorithm \#2 & Algorithm \#3 & Algorithm \#4 \\
\hline Machine \#1 & 350.321 & 235.810 & 3.96 & 0.15 \\
Machine \#2 & 276.823 & 200.578 & 3.647 & 0.073 \\
\hline
\end{tabular}

219

220

\section{Discussion}

\subsection{Performance}

TPR algorithm can help speed up the process of OBIA and shorten the interval between the image acquired date and the image analyzed date. A direct impact on service providers and endpoint users is the data processing time is reduced. There are three major modes for RS image analysis: manual, semi-automatic and automatic. The semi-automatic mode allows image analysts to use GIS/RS software such as ArcGIS, ENVI, ERDAS, eCognition for automatic assistance and is the most used one. The workflow is sequential. Once an analyst submits a request to the systems, (s) he will have to wait until the segmentation is completed. If one process like the pixel sorting process becomes faster, the time that analyzers spend on waiting will decrease respectively. The time for analyzing an image will lower too. Eventually the interval between the image acquiring date and the date when endpoint users receive the analyzed products will shrink. This is very meaningful in urgent cases such as earthquake, flooding, wild fire, debris flow and typhoon when every second counts.

\subsection{Application Vision}

This algorithm provides a good example for researchers to develop optimal complexity algorithms for time saving purposes. Most current researchers in RS mainly focus on improving result accuracy and show less care on time costs. In many researches, the efficiency of algorithms is sacrificed to pursue for a more accurate result such as Neural 
Network and SVM based image analysis techniques. Their outputs may have higher accuracy but take much longer than basic analysis techniques. In other words, more recent researches are engaged in augmenting the information value of RS products but ignore the time value. This work try to display the power of time-efficient algorithms in RS and make the overall duration of an analysis on a RS image into an endurable level.

\section{Conclusion and Future Work}

This paper proposes a novel algorithm, named TPR algorithm, to speed up the process of sorting pixels in segmented RS images. It avoids most multiple-layer loops in existing methods and trims many redundant comparison among pixels. It fully leverages the boundaries of vector features and uses two direction comparison to prevent duplicated comparison. Its Big $\mathrm{O}$ algorithm complexity is very lower than existing algorithms. We implemented the algorithm in $\mathrm{C}++$ and published the code onto GitHub. Experiments have been made by running the code with a high resolution optical RS test image on two different machines. In every single test, the time cost of TPR algorithm is always the lowest. The results prove that the new algorithm significantly decreases the time cost of the process. The average duration is reduced from 3.96 seconds to 0.15 second on machine \#1 and from 3.647 seconds to 0.073 second on machine $\# 2$. The algorithm sets a good example for time-efficient algorithms to speed up the overall process of OBIA in the big data era.

To make TPR algorithm able to serve as a robust building block in OBIA, we will maintain and update the code on GitHub. Besides, the current test image is a high resolution optical image. We will apply the algorithm on high spectral images in our next stage of work. In addition, how to fasten the other steps in OBIA while keeping the accuracy not going down 
260 will be studied too.

261

262

263 


\section{Reference}

1 Blaschke, T.: 'Object based image analysis for remote sensing', ISPRS Journal of Photogrammetry and Remote Sensing, 2010, 65, (1), pp. 2-16

2 Di, L., Yue, P., and Sun, Z.: 'Ontology-supported complex feature discovery in a web service environment', in Editor (Ed.) ${ }^{\wedge}(E d s$.$) : 'Book Ontology-supported complex feature$ discovery in a web service environment' (2012, edn.), pp. 2887-2890

3 Sun, Z., Fang, H., Di, L., Yue, P., Tan, X., and Bai, Y.: 'Developing a web-based system for supervised classification of remote sensing images', GeoInformatica, 2016, pp. 1-21

4 Sun, Z., Di, L., Chen, A., Yue, P., and Gong, J.: 'The use of geospatial workflows to support automatic detection of complex geospatial features from high resolution images', in Editor (Ed.)^(Eds.): 'Book The use of geospatial workflows to support automatic detection of complex geospatial features from high resolution images' (IEEE, 2013, edn.), pp. 159-162

5 Sun, Z., Di, L., and Fang, H.: 'Using long short-term memory recurrent neural network in land cover classification on Landsat and Cropland data layer time series', International Journal of Remote Sensing, 2018, 40, (2), pp. 593-614

6 Sun, Z., Di, L., Fang, H., Burgess, A.B., and Singh, N.: 'Deep Learning Cyberinfrastructure for Crop Semantic Segmentation', in Editor (Ed.)^(Eds.): 'Book Deep Learning Cyberinfrastructure for Crop Semantic Segmentation' (AGU, 2019, edn.), pp.

7 Duro, D.C., Franklin, S.E., and Dubé, M.G.: 'A comparison of pixel-based and object-based image analysis with selected machine learning algorithms for the classification of agricultural landscapes using SPOT-5 HRG imagery', Remote Sensing of Environment, 2012, 118, pp. 259-272

8 Blaschke, T., Hay, G.J., Kelly, M., Lang, S., Hofmann, P., Addink, E., Queiroz Feitosa, R., van der Meer, F., van der Werff, H., van Coillie, F., and Tiede, D.: 'Geographic Object-Based Image Analysis - Towards a new paradigm', ISPRS Journal of Photogrammetry and Remote Sensing, 2014, 87, pp. 180-191

9 Benarchid O., Raissouni N., El Adib S., Abbous A., Azyat A., Ben Achhab N., Lahraoua M. , and A., C.: 'Building Extraction using Object-Based Classification and Shadow Information in Very High Resolution Multispectral Images, a Case Study: Tetuan, Morocco', Canadian Journal on Image Processing and Computer Vision, 2013, 4, (1), pp. 1-8

10 Hussain, M., Chen, D., Cheng, A., Wei, H., and Stanley, D.: 'Change detection from remotely sensed images: From pixel-based to object-based approaches', ISPRS Journal of Photogrammetry and Remote Sensing, 2013, 80, pp. 91-106

11 Sun, Z., Fang, H., Deng, M., Chen, A., Yue, P., and Di, L.: 'Regular Shape Similarity Index: A Novel Index for Accurate Extraction of Regular Objects from Remote Sensing Images', Geoscience and Remote Sensing, IEEE Transactions on, 2015, 53, (7), pp. $3737-3748$

12 Heipke, C., Madden, M., Li, Z., and Dowman, I.: 'Theme issue "State-of-the-art in photogrammetry, remote sensing and spatial information science", ISPRS Journal of Photogrammetry and Remote Sensing, 2016, (115), pp. 1-2

13 Chi, M., Plaza, A.J., Benediktsson, J.A., Zhang, B., and Huang, B.: 'Foreword to the Special Issue on Big Data in Remote Sensing', Selected Topics in Applied Earth Observations and Remote Sensing, IEEE Journal of, 2015, 8, (10), pp. 4607-4609 
$14 \mathrm{https} / /$ earthdata.nasa.gov/about, accessed 2016.5.16

15 http://landsat.usgs.gov/Landsat_Project_Statistics.php, accessed 2014.9.21

16

https://hyspeedblog.wordpress.com/2013/03/22/big-data-and-remote-sensing-where-does -all-this-imagery-fit-into-the-picture/, accessed 2016.5.16

17 Ma, Y., Wu, H., Wang, L., Huang, B., Ranjan, R., Zomaya, A., and Jie, W.: 'Remote sensing big data computing: Challenges and opportunities', Future Generation Computer Systems, 2015, 51, pp. 47-60

18 Li, S., Dragicevic, S., Castro, F.A., Sester, M., Winter, S., Coltekin, A., Pettit, C., Jiang, B., Haworth, J., Stein, A., and Cheng, T.: 'Geospatial big data handling theory and methods: A review and research challenges', ISPRS Journal of Photogrammetry and Remote Sensing, 2016, 115, pp. 119-133

19 Rathore, M.M.U., Paul, A., Ahmad, A., Chen, B.W., Huang, B., and Ji, W.: 'Real-Time Big Data Analytical Architecture for Remote Sensing Application', IEEE Journal of Selected Topics in Applied Earth Observations and Remote Sensing, 2015, 8, (10), pp. 4610-4621

20 Hay, G.J., and Castilla, G.: 'Geographic Object-Based Image Analysis (GEOBIA): A new name for a new discipline': 'Object-based image analysis' (Springer, 2008), pp. 75-89

21 Shi, J., and Malik, J.: 'Normalized cuts and image segmentation', Pattern Analysis and Machine Intelligence, IEEE Transactions on, 2000, 22, (8), pp. 888-905

22 Ahmad, A., and Dey, L.: 'A k-mean clustering algorithm for mixed numeric and categorical data', Data \& Knowledge Engineering, 2007, 63, (2), pp. 503-527

23 Ball, G.H., and Hall, D.J.: 'ISODATA, a novel method of data analysis and pattern classification', in Editor (Ed.) ${ }^{\wedge}$ (Eds.): 'Book ISODATA, a novel method of data analysis and pattern classification' (DTIC Document, 1965, edn.), pp.

24 Comaniciu, D., and Meer, P.: 'Mean shift: A robust approach toward feature space analysis', Pattern Analysis and Machine Intelligence, IEEE Transactions on, 2002, 24, (5), pp. 603-619

25 Fauvel, M., Benediktsson, J.A., Chanussot, J., and Sveinsson, J.R.: 'Spectral and spatial classification of hyperspectral data using SVMs and morphological profiles', Geoscience and Remote Sensing, IEEE Transactions on, 2008, 46, (11), pp. 3804-3814

26 Manjunath, B.S., and Ma, W.-Y.: 'Texture features for browsing and retrieval of image data', Pattern Analysis and Machine Intelligence, IEEE Transactions on, 1996, 18, (8), pp. $837-842$

27 He, D.-C., and Wang, L.: 'Texture features based on texture spectrum', Pattern Recognition, 1991, 24, (5), pp. 391-399

28 Yan, J., Lin, L., Zhou, W., Ma, K., and Pickett, S.T.: 'A novel approach for quantifying particulate matter distribution on leaf surface by combining SEM and object-based image analysis', Remote Sensing of Environment, 2016, 173, pp. 156-161

29 Shirke, S., Pinto, S.M., Kushwaha, V.K., Mardikar, T., and Vijay, R.: 'Object-based image analysis for the impact of sewage pollution in Malad Creek, Mumbai, India', Environmental Monitoring and Assessment, 2016, 188, (2), pp. 1-12

30 Yan, M., Blaschke, T., Tang, H., Xiao, C., Sun, X., Zhang, D., and Fu, K.: 'Using object-based analysis to derive surface complexity information for improved filtering of airborne laser scanning data', Frontiers of Earth Science, 2016, pp. 1-9 
35131 Papadimitriou, C.H.: 'Computational complexity' (John Wiley and Sons Ltd., 2003.

352 2003)

35332 Papadimitriou, C.H., and Steiglitz, K.: 'Combinatorial optimization: algorithms and 354 complexity' (Courier Corporation, 1982. 1982)

35533 http://www.gdal.org, accessed Mar 202015

356

357

$358 \quad$ Figures

359 Figure 1. A segmented RS image and some sample segments

360 Figure 2. The workflow of OBIA

361 Figure 3. Sorting pixels located in vectorized polygons

362 Figure 4. Example inputted image and vector parameters and variables

363 Figure 5. The inputs and output of the test image. (a) original image; (b) segmented image; (c)

364 vector file; (d) pixel-sorted image

365 Figure 6. The time costs of four algorithms on two machines

366

367 
369 Table 1. The ranges of time cost for the four algorithms (unit: second)

370 Table 2. The average time costs of the four algorithms on two machines (unit: second) 371 